$\mathrm{DOE} / \mathrm{ER} / 13590--10$

DE92 012668

\title{
STUDIES OF ATMOSPHERIC MOLECULES BY MULTIPHOTON SPECTROSCOPY
}

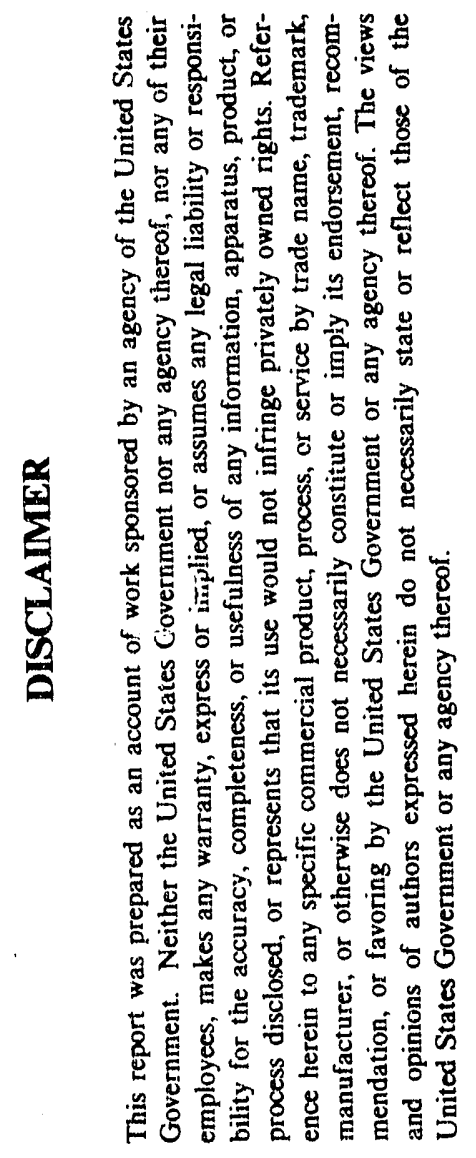

\author{
Progress Report \\ for Period July 15, 1989-October, 1991
}

Philip M. Johnson

Department of Chemistry

State University of New York

Stony Brook, NY 11794

October 1991

Prepared for

THE U.S. DEPARTMENT OF ENERGY AGREEMENT NO. DE-FG02-86ER13590 


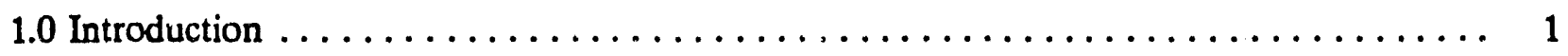

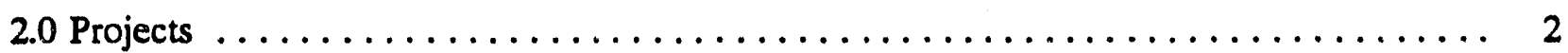

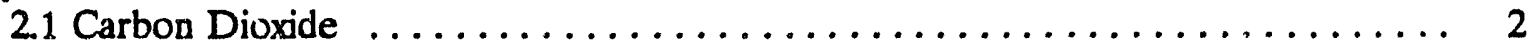

2.1.1 The $3+1$ multiphoton ionization spectrum $\ldots \ldots \ldots \ldots \ldots \ldots \ldots \ldots$

2.1.2 Autoionization structure and rotational contours $\ldots \ldots \ldots \ldots \ldots \ldots$

2.1.3 Photoabsorption above the ionization potential ........... 6

2.1.4 Competition between photoionization and dissociation $\ldots \ldots \ldots \ldots 8$

2.2 Threshold ionization spectroscopy of aromatics $\ldots \ldots \ldots \ldots \ldots \ldots \ldots \ldots \ldots \ldots 11$

2.2.1 Mass analyzed threshold ionization spectroscopy $\ldots \ldots \ldots \ldots \ldots \ldots 11$

2.2.2 Photophysical properties of pyrazine by threshold ionization

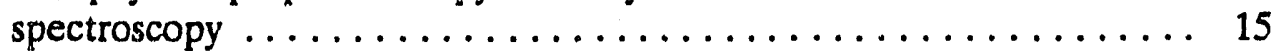

2.2.3 The vibrational structures of pyrazine and pyrimidine ions $\ldots \ldots \ldots \ldots 17$

2.3 The spectroscopy of the higher excited states of nitrogen $\ldots \ldots \ldots \ldots \ldots \ldots$

2.4 Collaborative efforts at Brookhaven National Laboratory . . . . . . . . . . . 18

2.4.1 The diode laser infrared spectroscopy of ethyl radical $\ldots \ldots \ldots \ldots \ldots 18$

2.4.2 VUV Photodissociation of Chlorofluorocarbons ............ 18

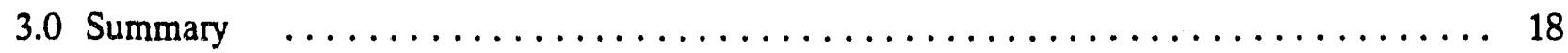




\subsection{Introduction}

During this grant period we have continued the development of laser ionization tools toward the study of the spectroscopy and dynamics of molecules relevant to combustion processes.

One of the molecules we have spent considerable effort on is carbon dioxide. Besides its obvious relevance in combustion, it presents a great challenge to spectroscopy because of its propensity toward dissociation in all of its excited states. Multiphoton ionization spectroscopy is usually not applicable to the study of dissociating molecules because the dissociation competes effectively with ionization, resulting in no signal. We reasoned, however, that with high enough laser fluence, ionization could compete with dissociation in the longer lived states, exposing them for study from the continuous spectral background resulting from rapidly dissociating states. This turned out to be possible, and in four papers we describe the various spectroscopic and photophysical effects found through the multiphoton ionization and multiphoton photoelectron spectra.

Photoelectron spectroscopy is a very useful technique for finding out about properties of both neutral molecules and ions. A recently developed variant of threshold ionization spectroscopy, usually called ZEKE, has shown a great deal of usefulness in providing the same information as traditional photoelectron spectroscopy (where the kinetic energy of ejected electrons are measured) but with higher resolution and much better signal-to-noise when using standard laboratory lasers. Threshold ionization techniques locate the states of an ion by scanning a light source across the ionization continuum of a neutral and somehow detecting when electrons are produced with no kinetic energy. It has recently been realized that the Z.EKE or pulsed field ionization (PFI) method of measuring threshold ionization spectra is exploiting the fjeld ionization of very high Rydberg states which exist just before each ionization threshold. Regardless of the: mechanism, the quality of spectra produced by the method is very high.

Aromatic molecules are of great importance to combustion since they constitute a significant fraction of unleaded gasoline. We chose to develop our capabilities in threshold ionization spectroscopy using aromatic molecules because of their importance and because their electronic structure allows a pump-probe type of excitation scheme which avoids the use of vacuum ultraviolet laser beams. Among aromatics, the azines are noted for their small $S_{1}-T_{1}$ energy gap which gives them unique and interesting photophysical properties. During this grant period we have studied the basic threshold ionization spectroscopy of diazines pyrazine and 
pyrimidine as a preliminary to the experiments proposed for the next grant period in which complexes of these molecules with reactive species such as oxygen are used to study oxidative mechanisms.

In the course of this work we have developed a new technique of detecting ionization thresholds which depends only upon measuring the ions produced and therefore retains mass information. This is done by exploiting the narrow kinetic energy distribution of the molecules in a supersonic beam and the fact that near a threshold ions can be separated from Rydbergs by a small electric field. With a series of grids in the beam path, field ionized Rydbergs are made to arrive at the detector before any directly produced ions and gating on the earlier arriving species while scanning ionization wavelengths produces a threshold ionization spectrum of only the mass of interest. The viability of the scheme has been demonstrated by producing the same spectrum for pyrazine by electron and ion detection. It is anticipated that this method will also enable the production of state selected ion beams for use in reactivity studies.

We have continued our work on the multiphoton spectrum of metastable nitrogen produc d by an electric discharge in a supersonic beam. We have been able to assign more of the lines and simulated their rotational structure but many peaks remain unassigned. Measurements of polarization and laser power dependences have strongly suggested that these peaks resulted from unidentified long-lived highly excited states lying in the energy above $100,000 \mathrm{~cm}^{-1}$.

Collaborative projects are underway at Brookhaven National Laboratory involving the infrared diode laser spectroscopy of ethyl radical with Trevor Sears, and the VUV photodissociation of chlorofluorocarbons with Michael White.

\subsection{Projects}

\subsection{Carbon Dioxide}

\subsubsection{The $3+1$ multiphoton ionization spectrum}

(J. Chem. Phys. 91, 7399 (1989))

In this initial study on this molecule, the MPI spectra of $\mathrm{CO}_{2}$ (figure 1) were recorded in the range 275 to $338 \mathrm{~nm}$ in bnth a conventional MPI cell and a molecular beam apparatus. The polarization ratio of each MPI peak was measured in order to assign the symmetry of excited states. The $3 \mathrm{p} \sigma_{u}{ }^{1} \Pi_{u}$ and $3 \mathrm{po}_{u}{ }^{1} \Pi_{u} 1_{0}^{1}$ Rydberg states were observed, while the $3 p \pi_{u}{ }^{1} \Sigma_{u}^{+}$Rydberg 
state was missing, and a new Rydberg state, $3 p \pi_{u}{ }^{1} \Delta_{u}$ was observed at 333.03 and 333.70 nm. In the $\mathrm{nf}(\mathrm{n}=4,5)$ Rydberg series sharp groups of resonances were seen which were not able to be assigned to any known states in the three photon region. A mass selected pure $\mathrm{CO}_{2}$ ion signal was measured for the $3 p \pi_{u}{ }^{1} \Delta_{u}$ state, but both $\mathrm{CO}^{+}$and $\mathrm{CO}_{2}^{+}$ion signals were detected for the other Rydberg states. From the power dependences of these ion products, it is suggested that the dissociation to produce $\mathrm{CO}^{+}$arises primarily in the ion and that neutral dissociation followed by ionization of $\mathrm{CO}$ is not a major channel.

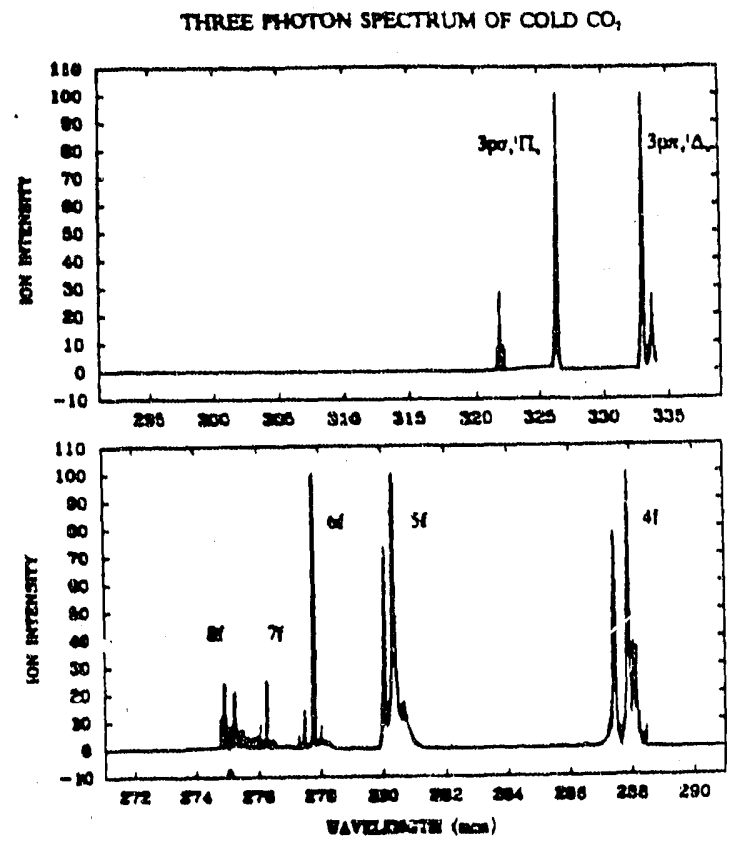

Figure 1

\subsubsection{Autoionization structure and rotational contours}

(J. Chem. Phys. 94, 868 (1991))

In the spectra reported in the initial $\mathrm{CO}_{2}$ study there appeared groups of very sharp lines associated with $\mathrm{f}$ Rydberg states which could not be assigned to bound resonances. These lines are much sharper than one would expect considering the rotational envelope of any expected three-photon resonances and appear in intriguingly simple patterns. $\mathrm{CO}_{2}$ is a novel molecule with respect to REMPI spectroscopy because almost all of its excited states are predissociative, and therefore few resonances are seen because dissociation competes effectively with ionization. However, the vacuum ultraviolet absorption spectrum is quite complex', with the Renner-Teller perturbed vibrational structure of several Rydberg series overlapping. In addition, spin-orbit splitting of the core energies and the broadening caused by pre lissociation create a situation in which individual features of the spectrum are not immediately assignable. The appearance of such sharp simple structure in the MPI spectrum of $\mathrm{CO}_{2}$ promiser to allow further insight into the excited state structure of a molecule in which only two of its transitions have been rotationally analyzed. ${ }^{2}$ 
In the measurement of resonance enhanced multiphoton ionization spectra we have become accustomed to interpreting the lines in the spectra in terms of multiphoton transitions to bound states of the atom or molecule. Usually the number of photons involved in ionizing an intermediate state is smaller than the number needed to excite it and the rate determining step in the ionization process is the initial excitation. The cross-section of the intermediate-to-continuum transition then drops out of the rate equations. ${ }^{3}$ Occasionally, particularly for atoms, there is the possibility of third harmonic generation ${ }^{4}$ which dramatically increases a three photon transition probability at the proper phase matching conditions and turns the ionization step into the limiting one. Then some continuum structure can be seen but it is rarely seen in the absence of third harmonic generation.

In order to understand the details of a resonant multiphoton ionization spectrum one must not only be able to anticipate the rotational, vibrational and electronic structure at the resonance level, but one must consider the contributions to the spectrum at every intermediate and virtual level along the route to ionization. The few resonances which are observed in the MPI spectrum of $\mathrm{CO}_{2}$ require relatively high amounts of laser power to see any signal in order for ionization to proceed on the same time scale as dissociation. Upon consideration of structure arising from all levels, it is found that this perturbation on the nonnal kinetic scheme allows continuumsuperimposed structure to appear in the spectrum with the same intensity as the bound resonances which are normally the only thing seen in MPI spectra.

Transitions to an $\mathrm{f}$ complex of $\mathrm{CO}_{2}$ can be very complicated, particularly with the selection rules of a three photon transition. The core of the state is ${ }^{2} \Pi_{z}$, which is split into $\Omega=1 / 2$ and $\Omega=3 / 2$ components with a separation of about $160 \mathrm{~cm}^{-1} .^{5}$ Each of these components is in turn split into four l-components, three of which are doubly degenerate. These l-components are partially coupled to the rotation axis instead of the internuclear axis, as is the spir of the Rydberg electron in an example of case-d Hund's coupling. Therefore strict $\Lambda$ and spin selection rules are not obeyed, giving rise to the possibility of much additional siructure. Because of the possibilities of structure arising from these complications, as well as Renner-Teller structure in the vibrations of the core and vibronic interactions with nearby Rydberg states, we were not able to assign the sharp structure accompanying the $4 \mathrm{f}$ and $5 \mathrm{f}$ resonances (figure 2 ). The other possibility, that the peaks were due to 4-photon resonances with autoionizing structure in the continuum, could only be considered upon the elimination of the possibility of resonances at the 3-photon level.

In order to anticipate the three photon spectral structure to be expected from an $\mathrm{f}$ - 
complex of a molecule with a ${ }^{2} \Pi$ core, it was necessary to calculate the rotational contour from first principles. Previous treatments of high-l complexes by Chang and Fano ${ }^{6}$ and by Herzberg and Jungen ${ }^{7}$ have dealt with sigma cores but provide the techniques for analysis of this type of spectrum. The open shell core of $\mathrm{CO}_{2}$ introduces complications associated with angular momentum of the core and spin-orbit coupling.

The spin and electronic

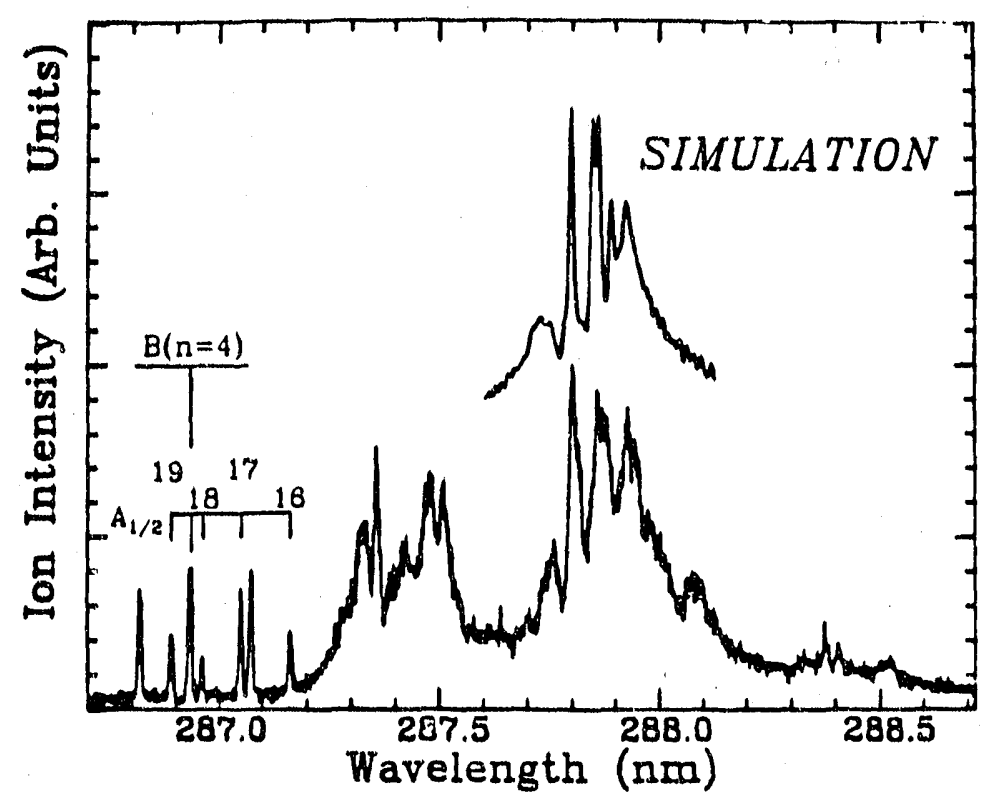

Figure 2 Room temperature MPI spectrum near the $4 f$ resonance. Calculated spectrum is shown above. angular momentum of the core electron must be considered to be tightly coupled to the molecular axis, while the Rydberg electron is allowed to uncouple its angular momenta from the molecular axis and instead be defined by the rotational axis in the laboratory frame. A given value of $J$, the total angular momentum, can be achieved in the molecule by various combinations of the rotation of the molecule, and the orbital angular momenta of the core and Rydberg electrons. Since the rotational energies are defined in the laboratory frame and the energies of the various electronic l-components as well as the electronic dipole matrix elements are defined in the molecular frame. in setting up a Hamiltonian matrix it is necessary to do a frame transformation of one set of energies into the other.

A program was written which included the proper frame transformation and calculated the rotational energy levels of a $\mathrm{CO}_{2}$ Rydberg state. Combining this with three-photon selection rules enabled the accurate simulation of the experimental spectrum using a very simple electronic energy model which assumed the electron was essentially uncoupled from the core axis.

Using this simulation program a reasonable facsimile to the broader spin-orbit split $f$ resonances could be obtained. All the details of the spectrum could not be reproduced, however. Under no choice of available parameters, including allowing interaction with d-orbitals and a full Renner-Teller vibrational treatment, were any structures produced which resemble the sharp 
peaks with no background to the blue of the $4 \mathrm{f}$ and $5 \mathrm{f}$ resonances. Lacking any explanation as 3photon resonances, resonances at the 4-photon level must be considered.

By considering the sharp structure to the blue of the $4 \mathrm{f}$ and $5 \mathrm{f}$ resonances to be structure in the continuum due to Rydberg series leading to the $\tilde{A}$ and $\tilde{B}$ states of the ion, most of the peaks are readily explainable, within the resolution of our experiment. Due to the higher density of states associated with $\bar{A}$, most of the peaks belong to series converging to various limits of that state. Since the $\tilde{A}$ series are only seen in the vicinity of $\ddot{B}$ Rydberg resonances, it appears that the oscillator strengths for the transitions are being provided by that configuration, although the lines assignable to $\tilde{B}$ resonances are not much stronger than the $\tilde{A}$ lines. Selection rules do not allow the peaks seen in the four photon spectrum to be the same ones seen in the one photon spectrum. Due to the proximity of the bound 3-photon $f$ resonances which enhance the 4-photon transitions, the autoiouizing Rydberg electrons are undoubtedly also in f orbitals, which differ little in energy from the $d$ orbitals in the one photon spectrum.

The overall process which produces the sharp multiphoton peaks can be viewed as the sequential excitation of an electron to an f Rydberg orbital followed by a core $\tilde{X}-\overline{\mathrm{B}}$ transition, producing a two-electron excited state in which the excited electrons are in such different orbitals that they do not interact. The $\tilde{\mathrm{B}}$ autoionizing level at the 4-photon level is strongly mixed with $\tilde{\mathrm{A}}$ Rydbergs of fairly high principal quantum number, creating a diffuse orbital with strong $l$ uscoupling. The long autoionization lifetime and the $l$-uncoupling produce the narrow bands which are so striking in the spectra.

\subsubsection{Photoabsorption above the ionization potential} (J. Chem. Phys. 94,7596 (1991))

One of the most remarkable effects seen in the high intensity irradiation of atoms and molecules is above threshold ionization (ATI). This phenomenon involves the absorption of photons by an atom or molecule even though the total energy contained in the various modes of the system is already greater than the ionization threshold. Experimentally it is usually detected by the sppearance of electrons with kinetic energies in excess of the difference between the ninimum number of photons energies required to exceed the ionization threshold and the energy of that threshold. This additional photon absorption usually proceeds because the electrons are kept localized in a ponderomotive potential created by the strong electric fields of an intense laser 
beam. ATI is commonly observed in atoms at intensities above $10^{11} \mathrm{~W} / \mathrm{cm}^{2}$ and this high intensity ATI has also been seen in hydrogen molecules by Cornaggia $e t a l^{8}$ and Verschuur et $a l^{9}$.

ATI should be able to be seen at much lower ficld strengths if the energy contained in the system were partitioned into different modes which were relatively uncoupled from one another. Then even though the total energy exceeds the ionization potential, the pooling of this energy into one electron may be slow enough to allow additional photons to be absorbed. Given the greater number of degrees of freedom, this phenomenon should be more prevalent in molecules and has been seen in nitric oxide by Miller and Compton ${ }^{10}$ and Kimman ei al $^{11}$. In that system there exist some vibrationally autoionizing states which are long lived because ionization can only occur through the transfer of several quanta of vibrational energy to a Rydberg electron. These long lived states are seen to absorb an additional photon at lower laser fluxes than are needed for ponderomotive confinement. We call this ionization through long-lived autoionizing states resonance enhanced above threshold absorption (REATA) to distinguish it from the ponderomotive force ATI mechanism.

In this work we made the observation of REATA proceeding through electronically autoionizing resonances of carbon dioxide. In general electronic autoionization is much faster than vibrational autoionization and states autoionizing by electronic interactions have not been previously observed to play a major role in REATA. However considerable REATA is seen in $\mathrm{CO}_{2}$ at intensities below $5 \times 10^{10} \mathrm{~W} / \mathrm{cm}^{2}$, a regime where the ponderomotive potential is not expected to be effective. Figure 3 shows the photoelectron spectrum ${ }^{12}$ resulting from the multiphoton ionization of carbon dioxide through an intermediate three photon resonance with the $4 \mathrm{f}$.Rydberg state. ${ }^{13}$ The four photon eneigy is above the lowest IP by $3.465 \mathrm{eV}$ but below any of the ionic electronic excited state energies. One would therefore expect to see only transitions to the $\mathrm{X}$ state of the ion, but there is also a band system at $1.994 \mathrm{Ev}$ which represents transitions to the $C$ innic state and requires that the molecule has absorbed 5 photons before ionization occurs.

For this particular resonance, the photon energy happens to be almost equivalent in the $\mathrm{X}$ to $B$ transition of the ion, so the picture is that of a three photon transition to the $4 f$ Rydberg level followed by a core excitation creating a two electron excited state. The Rydberg states leading to the $\mathrm{B}$ ionic state are strongly mixed with those leading to the $\mathrm{A}$ state ${ }^{\mathrm{I4}}$, which at the four photon level have principle quantum numbers around fourteen ${ }^{15}$. The state consisting of a diffuse Rydberg orbital combined with an excited core apparently has a long enough lifetime that 
ATI is possible at relatively low powers.

Using a typical absorption cross-section of $10^{-18} \mathrm{~cm}^{2}$ and the photon flux of this

experiment, a lifetime of greater than 50 ps can be estimated.

Similar results are obtained when the multiphoton excitation proceeds through the Sf Rydberg state at the three photon level, when the photon energy is almost resonant with the $\mathrm{X}(0,0,0)$ to $B(1,0,0)$ ionic transition. Another case of REATA is obtained through the $3 \mathrm{p} \sigma_{u}{ }^{1} \Pi_{u}$ state. Here the 5 photon level is below the ionic $\mathrm{C}$ state and photoelectrons are seen appropriate to transitions to various vibronic levels of the $B$ state. There are no clear two-electron resonances at the four photon level in this case, so the mechanism is not as obvious as in the other examples and will require a more rigorous theoretical analysis.

The type of REATA seen in $\mathrm{CO}_{2}$ should be a fairly general phenomenon, also occurring in atoms also because it is primarily electronic in origin. The existence of low lying ionic states which support Rydberg series within one photon energy of the first ionization potential increases the probability of accidental resonances, as does vibrational structure. A two color experiment, where one could tune separately to Rydberg and core transitions, should be able to produce REATA quite efficiently, and its presence could be used as a signature of the long lived two-electron excited states embedded in the electronic continuum.

\subsubsection{Compatition between photoionization and dissociation} (J. Chem. Phys. 95, 761 (1991))
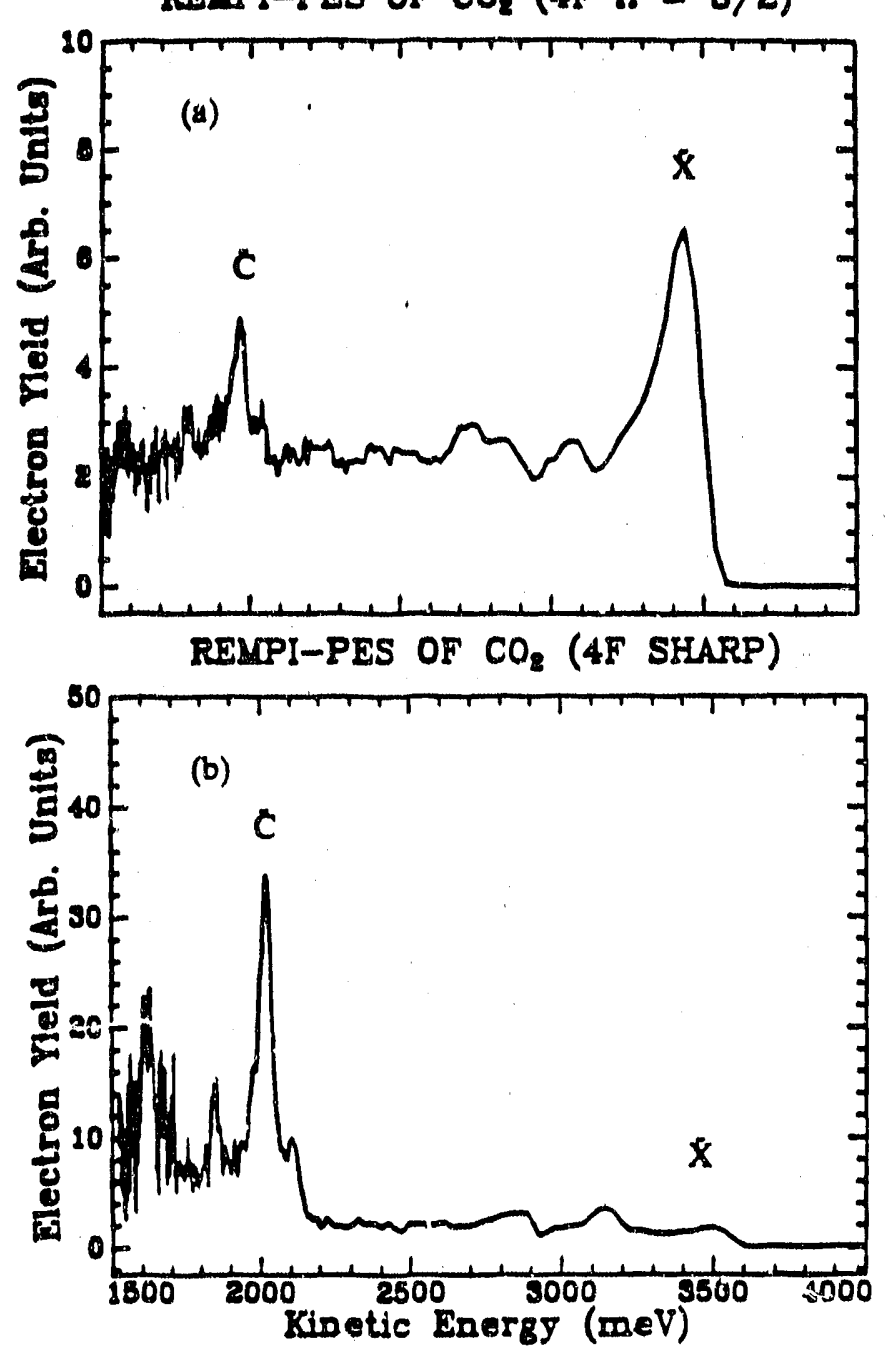

Figure 3 PES of $\mathrm{CO}_{2}$ : a) Resonant with the $4 \mathrm{f}$ state; b) Resonant with an autoionizing resonance. 
A long standing problem in chemistry is to understand the role of continuously evolving intermediate species. Technological advances, especially developments in lasers, have made it possible to probe the details of the transition states of molecules and extensive experimental and theoretical studies have appeared in the literature.

Absorption of transition species can be identified by observing spectral features which are not observed from either the reagents or products. In terms of dynamics, the absorption spectrum, $\epsilon(\omega)$ only describes the behavior in the local Franck-Condon region, and thus relates to a very short time scale. The width of $\epsilon(\omega)$ shows how fast the wave packet moves out of the Franck-Condon region. In this case, no information about the details of dynamics of the system can be addressed.

Light emission which takes place while a chemical bond is forming or breaking contains a great deal of information about molecular dynamics. The light emission can result from a iransition into either the ground-state continuum or the discrete vibronic eigenstates. The emission terminating on the ground-state continuum is expected to be broad and relatively featureless, although dynamical information can be obtained from studies of line shapes which are significantly affected by the variation of the transition moment with internuclear separation. When the emission terminates on discrete states, relative intensities of the various transitions, ie., the Franck-Condon overlaps, reflect the dynamics of the wave packet as it moves on the upper surface.

As an alternative to the use of light emission, REMPI-PES (Resonance Enhanced Multiphoton Ionization - Photoelectron Spectroscopy) can be extended to study a molecule undergoing ionization through a dissociative excited state. The behavior of the wave packet on the resonant intermediate surface will affect the Franck-Condon overlap between the excited and ionic states. Compared with light emission, $\operatorname{REMPI}^{16,17}$ is a complementary experimental scheme which is important because of its high sensitivity, capability to prepare high lying excited states, and ability to detect states which do not fluoresce. In REMPI, there is control over the steps in the process by variation in the intensity of the laser, unlike processes which depend upon spontaneous emission. One of the motivations of this work was to examine a system in which ionization could compete with dissociation.

The experimental resuits of an REMPI-PES study of the $3 p$, $4 f$ and $5 f$ Rydberg states of $\mathrm{CO}_{2}$ indicate that direct ionization is possible while the molecule is in the process of undergoing a unimolecular dissociation, resulting in long vibrational progressions being cbserved in 
photoelectron spectra from Rydberg states.

When the laser wavelength was tuned to the $3 p \sigma_{u}{ }^{1} \Pi_{u}$ state three photon resonance (figure 4), complicated vibrational structure was observed. In addition to a strong origin, symmetric stretching progressions were seen built upon even multiple quanta of the asymmetric stretch. The laser intensity required to observe the additional structure for the $3 \mathrm{p} \sigma_{v}{ }^{1} \Pi_{u}$ state is lower than that for the $3 \mathrm{p} \pi_{u}{ }^{1} \Delta_{u}$ states.

The $(3+1)$ ionization process through 3p Rydberg states to the $\bar{X}^{2} \Pi_{8}$ ionic state can be described as that of a d-like $(\mathrm{g})$ valence electron which is

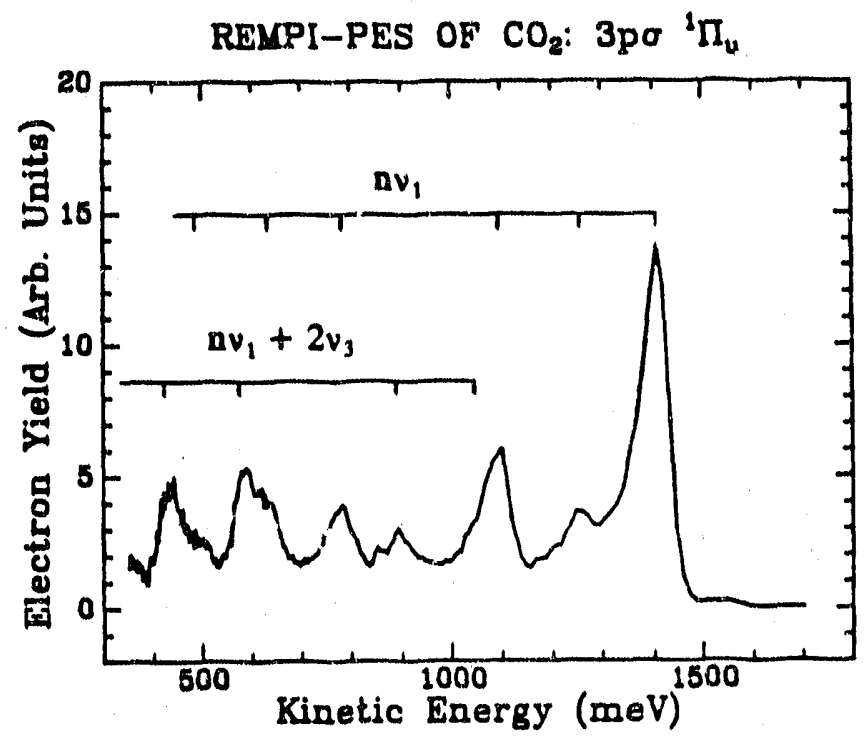
excited to a p-type (u) Rydberg orbital and then ionized. The outgoing

Figure 4

photoelectrons must be $\mathrm{g}$ and their wavefunctions will be dominated by $\mathrm{s}$ and $\mathrm{d}$ waves. Since both the neutral and ionic ground states are $\mathbf{g}$, the final electronic state (including the continuum) is $\mathbf{g}$ and a four photon process can only ocrur between vibrational level of the same inversion symmetry. Therefore in a four photon transition from the neutral vibrationless ground state, the final ionic state may have any number of quanta in the symmetric stretching mode, but only zero or an even number in each asymmetric or degenerate bending mode. The above assignments agree with these selection rules. It is possible to get an outgoing $u$ wave from a four photon ionization, but this would be a vibronicaliy induced process in this case and expected to be weaker.

It is interesting to note that the photoelectron intensity distributions seem to have two components. The first consists of $\tilde{X}^{2} \Pi_{B}(0,0,0)$ and $(1,0,0)$ transitions which are similar to those seen in one-photon photoelectron spectra and expected for a Rydberg resonance. The second component consists of several combination transitions of two quanta of the asymmetric stretching mode. These transitions have a different $F_{1}$ anck-Condon overlap which requires a relaxed geometry of the resonant excited state with respect to the ground ionic state. This implies that 
there may be two different competing ionization mechanisms in the MPI process.

The $(3+1)$ MPI structure of the 4 complex consists of two spin-orbit components split by $160 \mathrm{~cm}^{-1}$, and a series of broad peaks due to rotational structure and the various 1 -members of the f complex. The photoelectron

spectra (figure 5) of the ${ }^{2} \Pi_{23 / 2} 4 f$

Rydberg states ai $\lambda=287.82 \mathrm{~nm}$ (near the origin) and $287.77 \mathrm{~nm}$ (higher up in the rotational/1complex envelope) are almost the same, except that the $X(4,0,0)$ peak was observed only at the ${ }^{2} \mathrm{I}_{\mathrm{z}, 3 / 2} 4 \mathrm{f}$ origin and the $\mathrm{X}(2,0,2)$ peak was measured only in its component at $\lambda=287.77 \mathrm{~nm}$. The spectra of ${ }^{2} \Pi_{k 1 / 2}$ ff Rydberg states have the same structures at the two points $\lambda=287.38 \mathrm{~nm}$ and $287.25 \mathrm{~nm}$ in the rotational envelope .

When an intermediate state predissociates or mixes with a repulsive state, the violation of the $\Delta v=0$ propensity rule for MPI-PES is caused by a wave packet moving along its dissociation coordinate.

In our experimental results, a long asymmetric vibrational progression in the $\bar{X}^{2} \Pi_{g}$ state was obtained when in resonance with the af Rydberg series, although it does not appear in the single photon spectra. ${ }^{18,19,20,21,22,23,24}$

The most plausible interpretation of the photoelectron spectra present here suggests that ionization during molecular dissociation is responsible for the features of the spectra. In this model the unusually long progressions of asymmetric vibrations are caused by the molecules moving along the linear dissociation coordinate, which contains contributions from both the asymmetric and symmetric stretching modes. It should be noted that different resonances produce photoelectron spectra with different vibrational patterns. For example in the $3 p$ resonant spectra there are long symmetric progressions, while for the f resonances, asymmetric stretches dominate. 
If it were possible to model these spectra on a detailed level, these different vibrational intensities must contain information about the path of the reaction coordinate on the potential energy surface.

Linewidth-based estimates of the lifetimes of the $3 p$ Rydberg states vary between 0.2 and $0.3 \mathrm{ps}$, although this may be an underestimate because of rotational broadening. Similar estimates of the lifetimes of the $\mathrm{nf}$ states can be obtained from the rotational linewidths necessary to fit the rotational envelopes of those resonances. This is about $6 \mathrm{ps}$ for the $4 \mathrm{f}$ state and slightly shorter for the 5f. REMPI through these axcited states of $\mathrm{CO}_{2}$ must proceed on a time scale comparable to this lifetime if ionization is to compete with dissociation.

A simple kinetic model for the ionization and dissociation process has been previously presented. The validity of this model cannot be verified but the insertion of reasonable estimates for cross-sections, lifetimes, and laser power produces a consistent picture for the occurrence of competition. A $3+1$ ionization process is the best place to look for this phenomenon because the low probability of the three photon transition allows high intensities to be used witisut saturation. These high intensities are necessary in order to ionize the molecule on its journey down the reaction coordinate. Changing the intensity of the laser changes the dynamics of the process. We would expect, for example, that the vibrational envelopes in the photoelectron spectra would narrow up as the intensity is decreased. Unfortunately in a fourth order process with dissipative channels at several photon levels, the signal drops off very rapidly with laser power.

\subsection{Threshold ionization spectroscopy of aromatics}

\subsubsection{Mass analyzed threshold ionization spectroscopy}

(J. Chem. Phys. 94, 5769 (1991))

We have developed a new mass selective method for the determination of the optical spectra of the lower states of molecular ions and for the production of state selected ions. This method is similar to zero kinetic energy (ZEKE) photoelectron spectroscopy, but being mass selective, is applicable to systems such as clusters, radicals, van der Waals complexes, etc where species identification is necessary.

The spectroscopy of ionic molecular species is conveniently done by the observation of transitions from states of the neutral molecule. The most common method of obtaining spectra in this way involves measuring the kinetic energy of the photoionized electron, resulting in a 
photoelectron spectrum. Somewhat equivalent but higher resolution spectra of the ionic states can be acquired by somehow detecting an ionization threshold when scanning a variable wavelength light source across the ionization ccatinuum. A signature of these thresholds is the production of near-zero energy electrons. Techniques for their detection involving sector energy analysis $^{25}$ or aperture definition ${ }^{26}$ were developed in the early 1970 's and called threshold jonization spectroscopy. More recently a new technique for the detection of thresholds was developed which apparently involves the field ionization of Rydberg states with very high principal quantum numbers. ${ }^{27}$ The discrimination against directly ionized electrons is done by introducing a delay (typically $1-2 \mu \mathrm{sec}$ ) between the light pulse and a pulsed electric field which has the dual function of field ionizing any high Rydberg states and sweeping the resulting electrons to the detector. The delay allows any free electrons to disperse to the walls before the Rydberg states are field ionized. ${ }^{28}$ Although this is called zero kinetic energy electron spectroscopy, it does not necessarily involve a high resolution measurement of the electron kinetic energies. The value of the threshold detection methods is that they have much bigher energy resolution than standard photoelectron spectroscopy and suffer less from background and calibration problems. ZEKE spectra are commonly obtained with rotational resolution. A drawback of threshold techniques is that they generally require tunable light in the extreme ultraviolet, although it is often possible and desirable to use multiphoton transitions to offse $t$ this requirement.

A general disadvantage of any spectroscopy involving the detection of electrons rather than ions is the absence of mass information. Therefore environments where there may be more than one ionizing entity can produce ambiguous or uninterpretable results. Examples of these difficult situations include cluster spectroscopy, van der Waals complex spectroscopy, and the analysis of the products of chemical reactions. Photoelectron-photoion coincident techniques, PEPICO, have been developed to enable the simultaneous detection of the thresholds and the mass of the ionizing species. ${ }^{29}$ However by their very nature, coincidence techniques suffer from very low data acquisition rates and are not suited for use with low repetition rate lasers. We have developed a new method, called mass analyzed threshold ionization (MATI) spectroscopy which only depends upon the measurement of ions, and therefore provides mass resolved spectral information at high data acquisition rates.

Like ZEKE, MATI spectroscopy takes advantage of the field ionization of long lived high Rydberg states to signal an ionization threshold. As one scans a light source through the ionization continuum of an atom or molecule, these high Rydberg states will only be produced in 
direct conjunction with ionization limits and provide a signature for the thresholds' existence. In ZEKE, one rejects direct ionization events by simply waiting until all electrons produced by them exit the ionization region before counting the near-threshold Rydberg species by field ionizing them with a very small $(<1 \mathrm{~V} / \mathrm{cm}$ ) electric field pulse. This will not work for ions because their relatively low mobility keeps the ions and Rydbergs intermixed for longer than the autoionization lifetimes of the latter. It is, however, possible to apply a small field $(<1 \mathrm{~V} / \mathrm{cm})$ to the sample which can enable the separation of ions from the near-threshold neutrals. We have found that such a small voltage does not overly affect the lifetimes of the high-n states, although it lowers the ionization limit by a small, known amount $\left(\sim 6(E)^{1 / 2} \mathrm{~cm}^{-1}\right) \cdot{ }^{30}$

The scheme we have used to provide the spectrum presented in figure 6 involves using the kinetic energy of the molecules in a supersonic beam. The beam is directed through a series of four grids which define regions with fields of $0.8,-2$, and $-60 \mathrm{~V} / \mathrm{cm}$ if the molecular beam direction is defined as the $z$-axis of the system. In this experiment a fixed frequency laser pumps the origin of $S_{1}$ of pyrazine and a second tunable laser promutes the molecules into the ionization continuum, creating both ions and also high-n states if the wavelength is right. This takes place between the first two grids (region I) of the spectrometer. Since the retarding potential in this region is substantial with respect to the kinetic energy of the molecules in the beam,
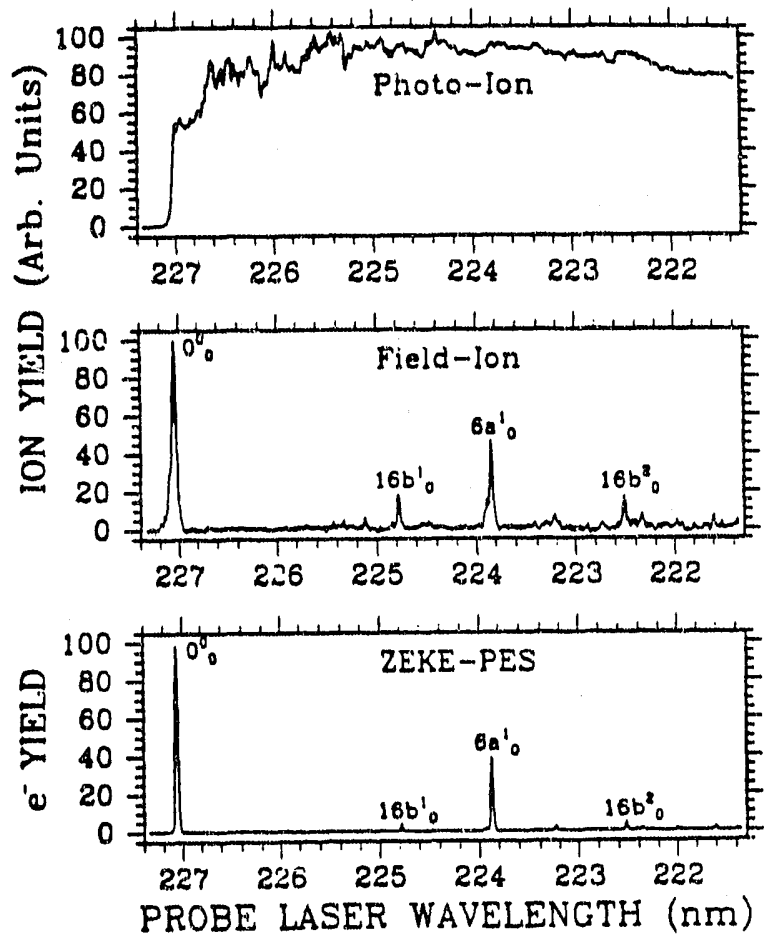

Figure 6 ZEKE, MATI, and total ion yield spectra of pyrazine through $0-0$ of $S_{1}$. the ions get slowed considerably before they get to the second grid, and by that tinse the ions have separated from the excited neutrals. Upon passing through the second grid (into region II) the near-threshold neutrals field ionize and are accelerated toward the third grid. Lower $\mathbf{n}$ states that do not field ionize fall behind and are separated by the time they reach the third grid, where they also field ionize. This provides improved wavelength resolution in the MATI spectra. All three groups of ions proceed into the flight region before arriving at the detector. The time of 
arrival distinguishes various masses and the different groups of each mass. In figure 6 , the photoion and MATI spectra were taken at the same time by monitoring the arrival of the directly ionized and the field ionized ions respectively. The difference in arrival times between the direct ions and the field ions was about $3 \%$ of the flight time, which constitutes five times the time difference between masses differing by one unit at the molecular weight of pyrazine. The ZEKE spectrum was taken in a separate apparatus using a standard delayed pulse extraction into a flight tube perpendicular to the molecular beam. The superior signal-to-noise of that spectrum is partially due to the fact that the ZEKE apparatus is much more fully developed.

Although we have not yet done so, it should be easily possible to apply retarding fields to the regions after the desired leading group has left, so that no signal is measured at the detector except when near-threshold Rydberg states are being created. This may be of particular advantage when trying to isolate a single mass from a mixture with closely spaced ionic weights. Transverse fields may also be advantageous in certain circumstances, but the present scheme has the virtue of simplicity.

The key parameter in this experiment is the electric field present in the ionization region. It is necessary that any Rydberg states have a long enough lifetime to travel from the ionization volume to the second grid, which is about $5 \mathrm{~mm}$ in our experiment. Therefore Rydbergs with very high $n$ are desirable since electrons in larger orbitals are less coupled to the vibrational and rotational motions of the core which can induce autoionization. Any voltage present in the ionization region lowers the threshold and polarizes the high-n orbits such that they have more interaction with the core. It is therefore desirable to reduce this voltage as much as possible and this will be the challenge of future experimental development. Of course any measured ionization limits must be corrected for the shift caused by the field present during ionization, while larger molecules may have lower signal to noise because of shorter autoionization lifetimes in the applied field.

The autoionization lifetime is dependent upon several things, such as the size of the ion core and the total amount of internal energy of the core. Our test molecule, pyrazine, is fairly large and provides a reasonable test of the technique. Smaller molecules should have greater lifetimes and therefore be less demanding concerning the applied voltages.

Wavelength resolution is dependent primarily on the difference between the electric fields in region I and region II. This determines how far past the depressed threshold the levels will be field ionized and thus the breadth of the wavelength response. Again, separation of ions and 
Rydbergs necessitates a certain minimum field level which will be a challenge to the design. Since autoionization in this region is detrimental, it is advantageous to keep region II short.

Besides enabling the spectroscopy of cluster ions, van der Waals complex ions, etc, the present scheme should also be useful for the creation of beams of state selected ions for use in reactivity and dynamics studies. Present techniques are either limited to molecules with appropriate Franck-Condon factors in an ionization process or rely on coincidence techniques with their inherent difficulties. MATI should allow state selected ion production with low repetition rate lasers, producing a substantial quantity of ions on each pulse.

The fact that this experiment is able to work lends strong support to a field ionized Rydberg mechanism as being the operable one in ZEKE. In fact, with the possible addition of some magnetic shielding, one should be able to do ZEKE in the same apparatus simply by reversing the voltages. The two types of detection should give the same spectra. However because of the bigher voltages which may be necessary for MATI, electron detection may have an advantage when the highest optical resolution and/or signal to noise is required. When mass information is desirable, MATI will be the method of choice and for many systems of current interest should provide spectroscopic information which would be difficult to obtain otherwise.

\subsubsection{Photophysical properties of pyrazine by threshold ionization spectroscopy}

$$
\text { (J. Chem. Phys. 95, } 2237 \text { (1991)) }
$$

Pyrazine has long served as a model in the development of ideas concerning the process of Intersystem Crossing (ISC), as has been recently reviewed by Kommandeur et al. ${ }^{31}$ In pyrazine, the $S_{1}-T_{1}$ gap is small enough that there are a limited number of zero order trijlet states isoenergetic to a given singlet level and therefore the singlet-triplet interaction should be describable without reference to a statistical approach. $T_{1}$ is coupled to $S_{1}$ by the spin-orbit operator to produce a set of mixed singlet-triplet states, or molecular eigenstates (ME's). In a typical low-resolution experiment, an optically prepared state can be envisioned as a linear combination of molecular eigenstates (LCME) evolving in time and therefore changing the singlet and triplet contributions in the mixed state. These mixed states are representative of a class of molecules which are intermediate with respect to their radiationless transition characteristics. In contrast to the statistical or large molecule limit in which the optically prepared state is composed of a large density or quasicontinuurn of coupled states, in an intermediate case molecule an 
optically prepared state is composed of a finite number of molecular eigenstates which have energy widths less than the energy separation between them.

The first experimental evidence of the existence of molecular eigenstates in pyrazine was obtained by van der Meer et al..$^{32}$ In their high resolution spectrum, individual $S_{1}-S_{0}$ rotational lines were shown to consist of many discrete lines of very narrow bandwidth. Later studies showed that the lifetimes, as well as the fluorescence quantum yields varied greatly among the ME's. ${ }^{33,}$ 43, 48,33,34,35,36

The number of lines within each rotational line was found to be too low in comparison to state counting ${ }^{37}$ and independent of $\mathrm{J}^{\prime},{ }^{33,43,48,38}$ contrasting with earlier time domain experiments which had shown a variation of quantum beats ${ }^{39,40,41}$ and decay characteristics ${ }^{42}$ with $\mathrm{J}^{\prime}$. The nature of this discrepancy has been recently discussed by Amirav ${ }^{43,44,45}$ based on a comparison of the rotational linewidths in the absorption and emission spectra. In his relatively low resolution experiments ( $2 \mathrm{GHz}$ ), a background absorption was detected between the rotational lines which dic not appear in the fluorescence excitation spectrum. Using a model first described by Matsumoto et al. ${ }^{46}$ the mixed singlet-triplet states are categorized into two groups. States with $\mathrm{S}_{1}-\mathrm{T}\left(\mathrm{J}^{\prime \prime}, \mathrm{K}^{\prime \prime}=0\right)$ have no Coriolis coupling and approximate the small molecule limit. These states are observed in the molecular eigenstate spectrum and have nanosecond lifetimes. Coriolis coupling within the triplet manifold is responsible for the dynamics of states with $S_{1}$ $\mathrm{T}\left(\mathrm{J}^{\prime \prime}, \mathrm{K}^{\prime \prime} \neq 0\right)$. A rotational IVR induced by Curiolis coupling redistributes the singlet component oscillator strength and therefore these states are only weakly observed in the absorption spectrum, have picosecond lifetimes, and produce a broad continuous absorption. According to Amirav, ${ }^{12.14}$ this explains the difference in rotational linewidths between the absorption and emission spectra.

However, other workers have not been able to detect any absorption between the clusters which comprise rotational lines in high resolution spectra. ${ }^{47}$ There is, however, considerable absorption within the clusters which does not show up in the fluorescence excitation spectra. It has also bcen found ${ }^{34}$ that $\mathrm{K}^{\prime}$ is a good quantum number for the ME's in the high resolution fluorescence excitation spectra, precluding $\mathrm{K}$ mixing in the triplet manifold for those levels, and that the $K^{\prime}=0$ levels have weaker fluorescence than the $K^{\prime} \neq 0$ leveis, in contrast to what would be expected from Amirav's model.

Previous experiments have yielded no information concerning the dynamical nature of the triplet componen! in the mixed states. Although it has been speculated that additional 
nonradiative processes (other than ISC) are in effect for the zero order triplet states, $44,45,44,45$ there has been no conclusive evidence to support this proposition. There have been no experiments designed to measure the redistribution of energy within the triplet manifold, which is thought to be caused by a rotationally induced IVR.44, 45,44, 45 The goal of this work was focused upon the description of the dynamics of the triplet component of the superposition state. Unlike fluorescence spectroscopy which is sensitive only to the singlet component of a mixed state, photoelectron transitions are not constrained by spin selection rules. By ionizing the triplet component of the mixed state, the triplet-ion Franck-Condon factors can provide information about $T_{1}$. If vibrational structure were to be resolved, it would be possible to identify the vibrational nature of the triplet component. However for pyrazine the density of states at 4056 $\mathrm{cm}^{-1}$ above the state origin of both the triplet state and the ion is such that resolution does not occur. Nevertheless the photoelectron spectrum provides information about the vertical ionization potential of the states located at $4056 \mathrm{~cm}^{-1}$ above the $T_{1}$ origin and allows the examination of the lifetimes of the subsets of triplet states optically coupled to various parts of the ionic vibrational manifold.

The pump-probe Pulsed Field Ionization (PFI) spectrum through the origin of $S_{1}$ of pyrazine reveals three distinct types of peaks (figure 7). At low excess energy above the lowest ionization potential (IP) are sharp peaks with Franck-Condon factors representative of the singlet intermediate state. At ionization wavelengths $<208 \mathrm{~nm}$ the FranckCondon overlap allows for the observation of primarily the triplet component of the pumped superposition state. At this energy some resolved structure has a measured lifetime $>10 \mu$ s and is determined to be the result of

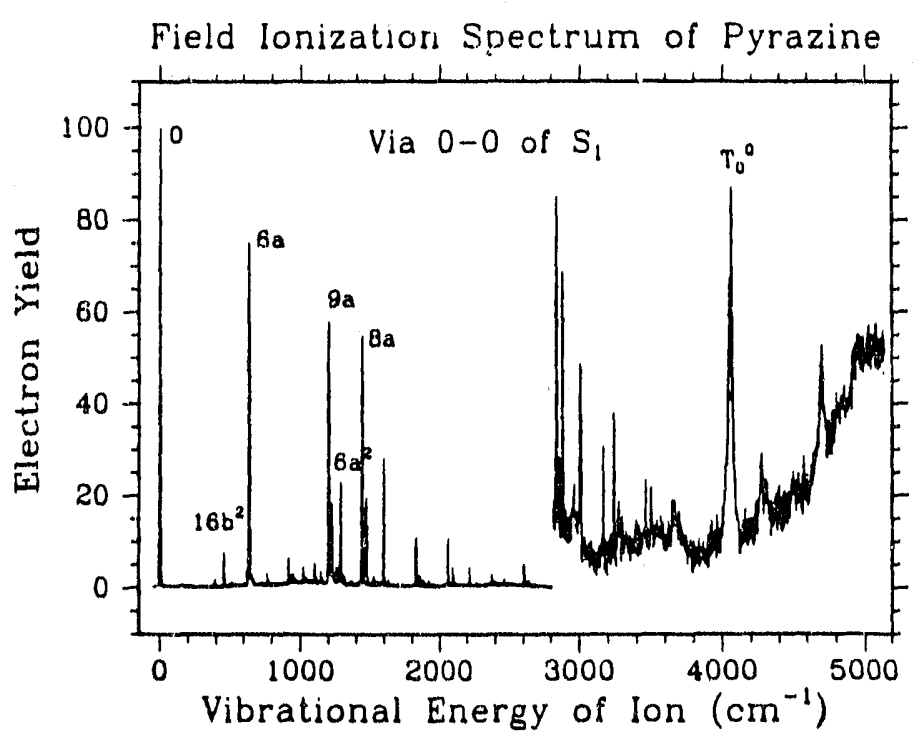
ionization of relaxed triplet states populated through vibrational dissociation of van der Waals 
clusters. A diffuse structure in the same region is the result of ionization of monomer intermediate states with lifetimes that vary with the ionizing wavelength. From this variation it can be surmised that thero is an inhomogeneous sample of intermediate states, possibly due to a mixture of unrelaxed molecular eigenstates and decoupled triplets populated by an intramolecuiar vibrational relaxation within the triplet manifold.

2.2.3 The vibrational structures of pyrazine and pyrimidine ions

The pump-probe threshold ionization spectra obtained through various intermediate resonances in $S_{1}$ provide a great deal of information about the vibrations of the terminating ionic grcund state. Figure 8 shows a couple of these spectra, indicating this wealth of information. We are in the process of analyzing these spectra, relying on vibrational frequencies derived from $a b$ initio calculations of the force fields of the ions. Upon completion of this analysis, we believe the result will be one of the most complete descriptions of the potential of a larger ion. This vibrational study will also form the basis for further work on the study of reactions of aromatic molecules in the gas phase.

2.3 The spectroscopy of the higher excited states of nitrogen

(MS thesis, M. Yoshuoka, USB department of chemistry, 1990)

We have continued our investigation of the long-lived states of nitrogen produced in a supersonic beam by an electric discharge. Work on the technique has improved the stability of the discharge and lengthened the life of the pulsed valve, both impediments in our previous work. New multiphoton ionization spectra of the metastables in the visible region were taken and a few bands were able to be analyzed and the rotational structure simulated. The metastables were identified as the $\mathrm{a}^{\prime}$ and the $\mathrm{E}$ states. Most bands remain a mystery however, and information from photoelectron spectroscopy will probably be necessary in order to determine the energies of the lower metastable states, which are shown to be near the ionization potential.

\subsection{Collaborative efforts at Brookhaven National Laboratory}

2.4.1 The diode laser infrared spectroscopy of ethyl radical

(with Trevor Sears) 
We have measured the infrared spectrum of ethyl radical with full rotational resolution between 490 and $550 \mathrm{~cm}^{-1}$ (with a few gaps). This was done by means of a new computercontrolled experimental system which uniquely measures the sample spectrum, a reference spectrum, and an etalon trace simultaneously. The general appearance of the spectrum fits well with a model which includes the internal rotation of the methyl group, but detailed assignments have not been forthcoming due to uncertainties in $\mathrm{J}$ numbering. A supersonic beam apparatus with a slit nozzle is being constructed for this experiment in order to provide cooled spectra which will enable the locations of the origins of the rotational series.

\subsubsection{VUV}
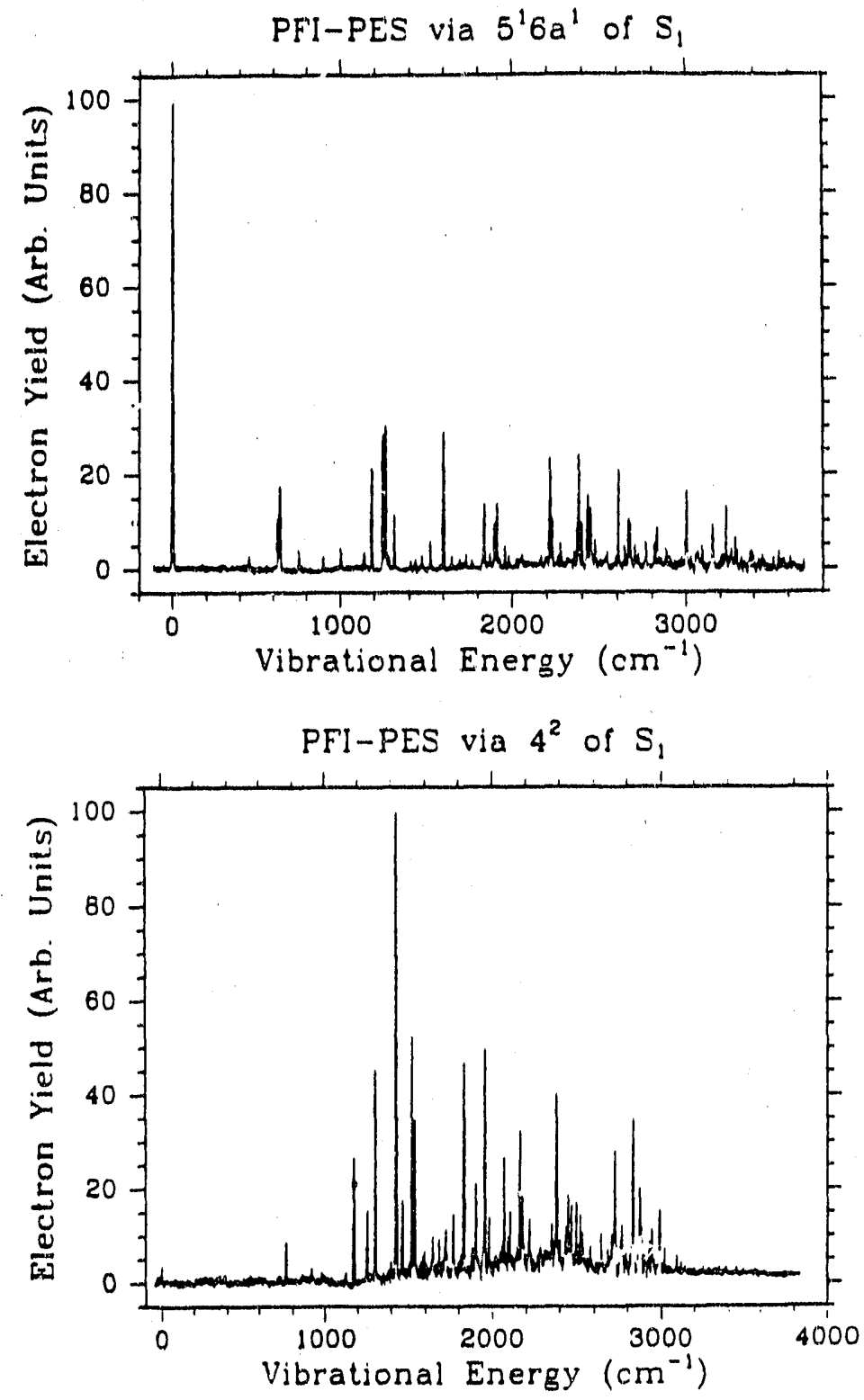

Figure 8 ZEKE spectra of excited vibrational levels of the $\mathrm{S}_{1}$ state of pyrazine.

Photodissociation of Chlorofluorocarbons

(with Michael White)

VUV photodissociation of $\mathrm{CF}_{3} \mathrm{Cl}$ has been studied by TOF (time-of-flight) photofragment spectroscopy. The VUV sources, i.e., $118 \mathrm{~nm}$ and $125 \mathrm{~nm}$, were generated by the third harmonic generation of $\mathrm{Xe}$ gas and the four-wave mixing of mercury vapor respectively. Atomic chlorine fragments in the ${ }^{2} \mathrm{P}_{1 / 2}\left(\mathrm{Cl}^{*}\right)$ spin states were detected at both wavelengths. The translational 
energies of the $\mathrm{Cl}$ fragments were measured from $(2+1)$ REMPI-TOF mass spectra. Two groups of the $\mathrm{Cl}$ tragments with different translational energies were found at $118 \mathrm{~nm}$ whereas there was only one at $125 \mathrm{~nm}$. The most probable center-of-mass translational energies released as a result of photodissociations were: $118 \mathrm{~nm}$ cissociation with $\mathrm{Cl}$ fragments: 1.15 and $0.23 \mathrm{eV} ; 125 \mathrm{~nm}$ dissociation with $\mathrm{Cl}$ fragments: $1.09 \mathrm{eV} ; 125 \mathrm{~nm}$ dissociation with $\mathrm{Cl}^{*}$ fragments: $1.15 \mathrm{eV}$. VUV photodissociation studies of other CFC molecules are under investigation.

\subsection{Summary}

Carbon dioxide turned out to be a gold mine of interesting spectroscopy and photophysical effects. We were able to record the three photon MPI spectrum and analyze it on the level of simulating the band contours, thus feeling confident that the spectroscopy is well in hand. This spectroscopic understanding enabled the recognition of other features such as resonances in the ionization continuum. The photoelectron spectra when resonant with the various states seen in the MPI spectrum added further interesting observations, including above threshold absorption and ionization during dissociation. This latter effect still presents a challenge with respect to getting detailed molecular information out of the spectra.

Our efforts on the threshold ionization spectroscopy of aromatic molecules, begun as an exploratory endeavor, resulted in clarification of some radiationless transition effects in pyrazine. More importantly, it led to the development of a major new experimental technique which we believe will be of general use as a tool for the spectroscopy of ions as well as neutrals and as a useful adjunct of mass spectrometry.

The PI spent a sabbatical year at Brookhaven National Laboratory in 1989-90. While there, collaborations were begun with both Trevor Sears and Michael White which are still continuing with substantial progress being made. This is greatly facilitated by the proximity of BNL and Stony Brook, as well as a fortuitous overlap of interests.

\section{References}

1. C. Cossart-Magos, J. Jungen, and F. Launay, Mol. Phys. 61, 1077 (1987).

2. R. N. Dixon, Proc. R. Soc. London Ser. A 275, 431 (1963). C. Cossart-Magos, S. Leach, M. Eidelsberg, F. Launay, and F. Rostas, J. Chem. Soc. Faraday Trans. 2 78, 1477 (1982). 
3. J.R. Ackerhalt and B. W. Shore, Phys. Rev. A16, 277 (1977). D. Zakheim and P. M. Johnson, Chem. Phys. 46, 263 (1980).

4. K. Aron and P. Johnson, J. Chern. Phys. 67, 5099 (1977). R. N. Compton and J. C. Miller, J. Opt. Soc. Am. B2, 355 (1984). L Li, M. Wuy and P. M. Johnson, J. Chem. Phys. 89, 3470 (1988),

5. J. M. Frye and T. J. Sears, Mol. Phys. 62, 919 (1987).

6. E. S. Chang and U. Fano, Phys. Rev. A 6, 173 (1972).

7. G. Herzberg and Ch. Jungen, J. Ch. 'a. Phys. 84, 1181 (1986).

8. C. Comaggia, D. Normand, J. Morellec, G. Mainfray, and C. Manus, Phys. Rev. A 34, 207 (1986).

9. J. Verschuur, L. Noordam, and H. van Linden van den Heuvell, Phys. Rev. A 40, 4383 (1989).

10. J. Miller and R. Compton, J. Chem. Phys. 75, 22 (1981).

11. J. Kimman, J. Verschuur, M. Lavollee, H. van den Heuvell, and M. van der Wiel, J. Phys. B 19, 3909 (1986).

12. This spectrum was taken in a permaner: magnet magnetic bottle instrument of our own design, but based upon the ideas of P. Kruit and F. Read, J. Phys. E 16, 313 (1983).

13. M. Wu and P. Johnson, J. Chem. Phys. 91, 7399 (1989).

14. J. Rostas and R. P. Tuckett, J. Mol. Spec. 96, 77 (1982).

15. Y. Tanaka and M. Ogawa, Can. J. Phys. 40, 879 (1962).

16. P.M. Johnson, Acc. Chem. Res., 13, 20 (1980)

17. P.M. Johnson and C.E. Otis, Ann. Rev. Phys. Chem., 32, 139, (1981)

18. F. Bueso-Sanllehi, Phys. Rev. 60, 566 (1941).

19. B. Kovac, JCP 78, 1684 (1983).

20. V. Kumar and E. Krishəakuman, JCP 78, 46 (1983).

21. I. Reineck, C. Hohre, R. Maripuu, P. Lodin, S.H. Al-Shamma, H. Veenhuizen, L. Karlsson and K. Siegbahn, Chem. Plays. 78, 311 (1983).

22. B. Wannberg, H. Veehhuizen, I. Mattsson, K.E Norell, L. Karlsson and K. Siegbahn, J. Phys. B, 17, L259 (1984). 
23. T. Baer and P. Guyon, J. Chem. Phys. 85, 4765 (1986).

24. L.S. Wang, J.E. Reutt, Y.T. Lee and D.A. Shirley, J. Electron Spectrosc. 47, 167 (1988).

25. D. Villarejo, R. Herm, and M. Inghram, J. Chem. Phys. 46, 4995 (1967).

26. T. Baer, W. Peatman, and E. Schlag, Chem. Phys. Lett. 4, 243 (1969).

27. IK. Müller-Dethlefs, M. Sander, and E. Schlag, Z. Naturforsch. 39a, 1089 (1984); Chem. Phys. Lett. 112, 291 (1984).

28. G. Reiser, W. Habenicht, K. Müller-Dethlefs and E. Schlag, Chem. Phys. Lett. 152, 119 (1988).

29. B. Brehm, and E von Puttkamer, Z. Naturforsch. 22a, 8 (1967).

30. C. Bordas, P. Brevet, M. Broyer, J. Chevaleyre, and P. Labastie, Europhys. Lett. 3, 789 (1987).

31. J. Kommandeur, W. Majewski, W. Meerts and D. Pratt, Ann. Rev. Phys. Chem. 38, 433 (1987).

32. B. van der Meer, H. Jonkman, J. Kommandeur, W. Meerts and W. Majewski. Chem Phys. Lett. 92, 565 (1982).

33. W. Siebrand, W. Meerts, and D. Pratt, J. Chem. Phys. 90, 1313 (1989).

34. W. Lawrance and A. Knight, J. Phys. Chem. 89, 917 (1985).

35. W. van Herpen, W. Meerts, K. Drabe and J. Kommandeur, J. Chem. Phys. 86, 4396 (1987).

36. A. Amirav and J. Jortner, J. Chem. Phys. 84, 1500 (1986).

37. P. Haarhoff, Mol. Phys. 7, 101 (1963).

38. B. van der Meer, H. Jonkman and J. Kommandeur, Laser Chem. 2, 77 (1983).

39. S. Okajima, H. Saigusa and E. Lim, J. Chem. Phys. 76, 2069 (1982).

40. P. Felker, W. Lambert and A. Zewail, Chem. Phys. Lett. 89, 309 (1982).

41. B. van der Meer, H. Jonkman, G. ter Horst and J. Kommandeur, J. Chem. Phys. 16, 2099 (1982).

42. G. ter Horst, D. Pratt, and J. Kommandeur, J. Chem. Phys. 74, 3616 (1981).

43. A. Amirav, J. Chem. Phys. 88, 2840 (1988). 
44. A Amirav, J. Phys. Chem. 92, 3725 (1988).

45. A. Amirav, Chem. Phys. 108, 403 (1986).

46. Y. Matsumoto, L. Spangler, and D. Pratt, J. Chem. Phys. 80, 5539 (1984).

47. W. van Herpen, P. Uijt de Haag, and W. Meerts, J. Chem. Phys. 89, 3939 (1989). 

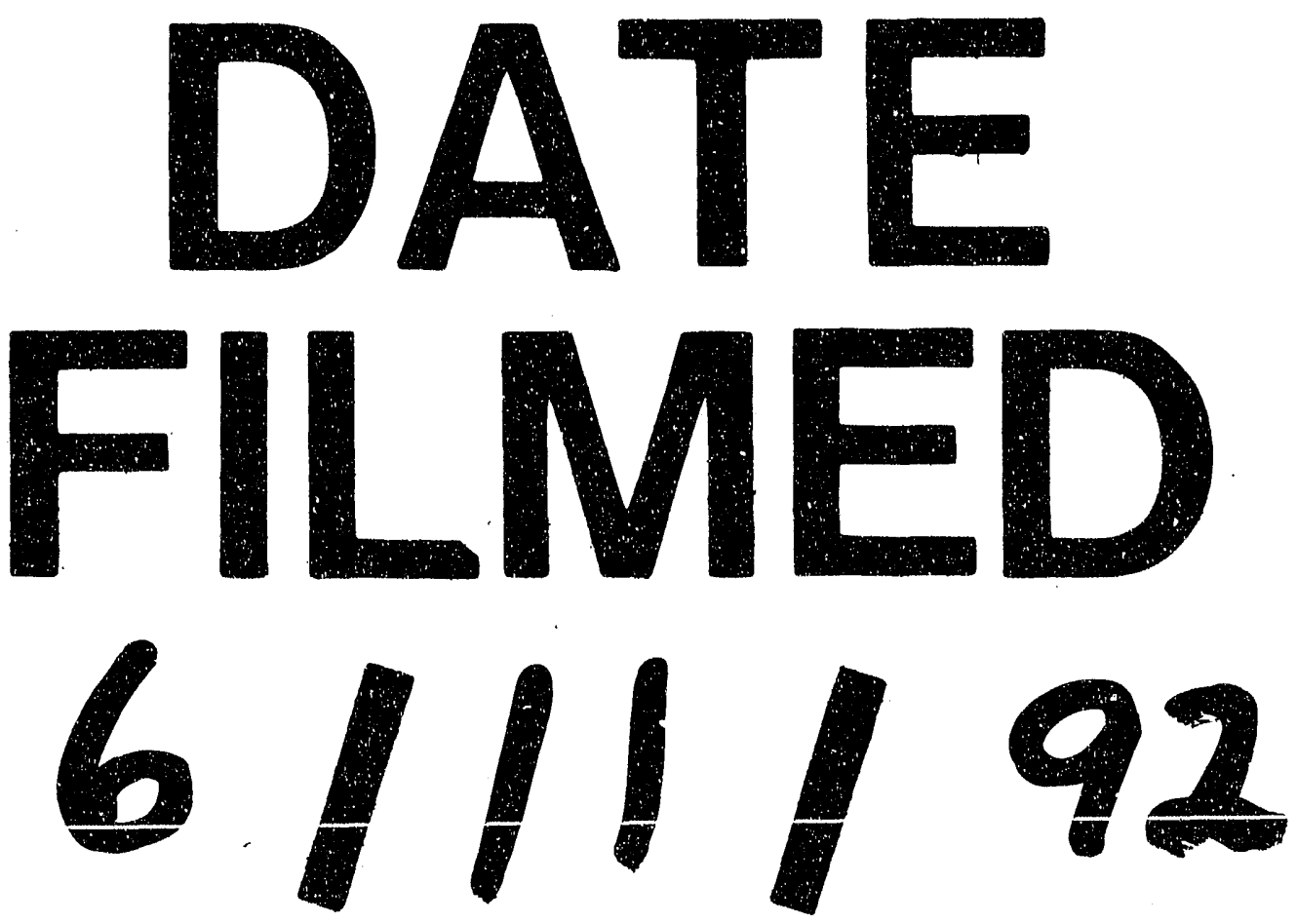


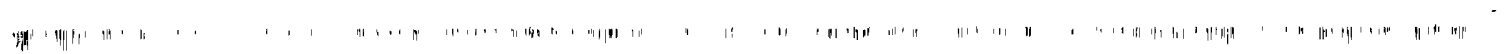

